\title{
The Anomeric Effect in Five-Membered Ring Molecules. Comparison of Theoretical Computations and Experimental Spectroscopic Results
}

\author{
Esther J. Ocola and Jaan Laane* \\ Department of Chemistry, Texas A\&M University, College Station, TX 77843-3255, USA \\ Institute for Quantum Science and Engineering, College Station, TX 77843-3255, USA
}

\section{SUPPORTING INFORMATION}

(This file includes twelve tables and three figures in fourteen pages.)

\section{Table of Contents}

Table S1. Structural Parameters for Molecules I to VI from CCSD/cc-pVTZ Computations.

Table S2. Structural Parameters for Molecules I to VI from MP2/cc-pVTZ Computations.

Table S3. Structural Parameters for VII from CCSD/cc-pVTZ and MP2/cc-pVTZ Computations.

Table S4. Structural Parameters for VII to XII from MP2/cc-pVTZ Computations.

Table S5. Coefficients of the Kinetic Energy Functions for Molecules I to XII.

Table S6. Comparison of Average Square Deviations (ASD) for the Frequency Fits of 1,3-Dioxole (I).

Figure S1. Calculated Ring-Puckering Energy Levels from (a) CSSD/cc-pVTZ Computations and (b) from MP2/cc-pVTZ Computations for 1,3-Dioxole (I).

Figure S2. Calculated Ring-Puckering Energy Levels from (a) CSSD/cc-pVTZ Computations and (b) from MP2/cc-pVTZ Computations for 1,3-Benzodioxole (VII).

Figure S3. Calculated Ring-Puckering Energy Levels from MP2/cc-pVTZ Computations for (a) VII, (b) VIII,

(c) IX, (d) X, (e) XI and (f) XII. The dashed line in (a) corresponds to the Experimental Fit for 1,3-Benzodioxole (VII).

Table S7. Calculated Ring-Puckering Transitions $\left(\mathrm{cm}^{-1}\right)$ for 1,3-Oxathiole (II).

Table S8. Calculated Ring-Puckering Transitions $\left(\mathrm{cm}^{-1}\right)$ for 1,3-Oxaselenole (III).

Table S9. Calculated Ring-Puckering Transitions $\left(\mathrm{cm}^{-1}\right)$ for 1,3-Dithiole (IV).

Table S10. Calculated Ring-Puckering Transitions $\left(\mathrm{cm}^{-1}\right)$ for 1,3-Thiaselenole (V).

Table S11. Calculated Ring-Puckering Transitions $\left(\mathrm{cm}^{-1}\right)$ for 1,3-Diselenole (VI).

Table S12. Calculated Ring-Puckering Transitions $\left(\mathrm{cm}^{-1}\right)$ from MP2/cc-pVTZ computations for VII-XII.

Corresponding author: Prof. Jaan Laane, laane@ chem.tamu.edu. 
Table S1. Structural Parameters for Molecules I to VI from CCSD/cc-pVTZ Computations

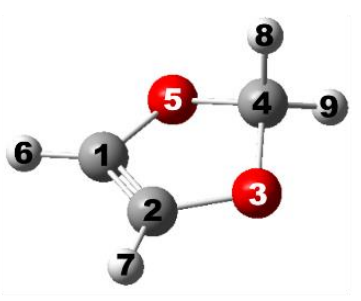

Molecule

\begin{tabular}{llllll}
\hline I & II & III & IV & V & VI \\
\hline
\end{tabular}

Bond lengths $(\AA)$

$\mathrm{C}_{1}=\mathrm{C}_{2}$

$\mathrm{C}_{1}-(\mathrm{O}, \mathrm{S}, \mathrm{Se}) \mathrm{C}_{5}$

$\mathrm{C}_{2}-(\mathrm{O}, \mathrm{S}, \mathrm{Se})_{3}$

$(\mathrm{O}, \mathrm{S}, \mathrm{Se})_{3}-\mathrm{C}_{4}$

$\mathrm{C}_{4}-(\mathrm{O}, \mathrm{S}, \mathrm{Se})_{5}$

$\mathrm{C}_{1}-\mathrm{H}_{6}$

$\mathrm{C}_{2}-\mathrm{H}_{7}$

$\mathrm{C}_{4}-\mathrm{H}_{8}$

$\mathrm{C}_{4}-\mathrm{H}_{9}$

1.327

1.330

1.330

1.333

1.332

1.331

1.385

1.371

1.368

1.766

1.765

1.896

1.385

1.775

1.903

1.766

1.897

1.896

1.418

1.835

1.969

1.822

1.954

1.951

1.418

1.420

1.418

1.822

1.819

1.951

1.073

1.077

1.078

1.079

1.080

1.080

1.073

1.075

1.075

1.079

1.079

1.080

1.097

1.092

1.090

1.088

1.086

1.085

1.085

1.085

1.084

1.086

1.086

1.085

Angles (degrees)

$\mathrm{C}_{1}=\mathrm{C}_{2}-(\mathrm{O}, \mathrm{S}, \mathrm{Se})_{3}$

$(\mathrm{O}, \mathrm{S}, \mathrm{Se})_{5}-\mathrm{C}_{1}=\mathrm{C}_{2}$

110.2

110.2

111.3

111.4

117.5

117.2

119.2

$\mathrm{C}_{2}-(\mathrm{O}, \mathrm{S}, \mathrm{Se})_{3}-\mathrm{C}_{4}$

103.1

$\mathrm{C}_{4}-(\mathrm{O}, \mathrm{S}, \mathrm{Se})_{5}-\mathrm{C}_{1}$

117.3

119.3

117.5

119.6

119.2

103.1

$(\mathrm{O}, \mathrm{S}, \mathrm{Se})_{3}-\mathrm{C}_{4}-(\mathrm{O}, \mathrm{S}, \mathrm{Se})_{5}$

107.1

86.7

82.9

93.3

89.9

92.4

108.7

110.7

93.3

107.8

107.7

108.9

95.6

92.4

131.9

126.3

125.3

124.2

109.1

109.3

131.9

127.9

126.8

124.2

123.7

123.3

117.9

122.2

123.2

118.2

123.6

123.3

109.2

109.2

108.7

119.0

117.5

$(\mathrm{O}, \mathrm{S}, \mathrm{Se})_{3}-\mathrm{C}_{4}-\mathrm{H}_{8}$

109.9

$(\mathrm{O}, \mathrm{S}, \mathrm{Se})_{3}-\mathrm{C}_{4}-\mathrm{H}_{9}$

109.2

110.7

109.8

109.5

110.0

$(\mathrm{O}, \mathrm{S}, \mathrm{Se})_{5}-\mathrm{C}_{4}-\mathrm{H}_{8}$

109.2

110.9

109.1

109.0

108.5

109.9

109.0

109.6

109.8

110.3

110.0

$(\mathrm{O}, \mathrm{S}, \mathrm{Se})_{5}-\mathrm{C}_{1}-\mathrm{H}_{6}$

117.9

114.8

108.7

109.1

108.7

108.5

111.6

113.9

118.2

116.8

117.5

110.9

111.1

110.1

110.3

110.4

\section{Angles between bonds}

$\mathrm{C}_{1}=\mathrm{C}_{2} /(\mathrm{O}, \mathrm{S}, \mathrm{Se})_{3}-\mathrm{C}_{4}$

$\mathrm{C}_{2}=\mathrm{C}_{1} /(\mathrm{O}, \mathrm{S}, \mathrm{Se})_{5}-\mathrm{C}_{4}$

15.2

$-15.2$

$(\mathrm{O}, \mathrm{S}, \mathrm{Se})_{3}-\mathrm{C}_{2} / \mathrm{C}_{1}-(\mathrm{O}, \mathrm{S}, \mathrm{Se})_{5}$

-15.2
0.0

15.4

14.4

$-19.1$

$-20.0$

18.4

16.8

16.5

$(\mathrm{O}, \mathrm{S}, \mathrm{Se})_{3}-\mathrm{C}_{2} /(\mathrm{O}, \mathrm{S}, \mathrm{Se})_{5}-\mathrm{C}_{4}$

$-1.2$

$-0.9$

$-18.4$

$-18.6$

$-16.5$

$-15.8$

$-19.5$

$-19.6$

0.0

0.3

0.0

15.8

16.9

16.5

$-19.8$

$-19.3$

$-17.7$

$(\mathrm{O}, \mathrm{S}, \mathrm{Se})_{5}-\mathrm{C}_{1} /(\mathrm{O}, \mathrm{S}, \mathrm{Se})_{3}-\mathrm{C}_{4}$

19.8

18.8

17.7 


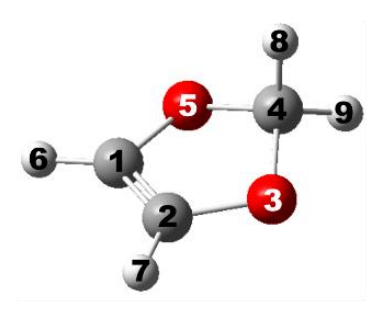

Molecule

\begin{tabular}{llllll}
\hline I & II & III & IV & V & VI \\
\hline
\end{tabular}

\section{Bond lengths $(\AA)$}

$\mathrm{C}_{1}=\mathrm{C}_{2}$

$\mathrm{C}_{1}-(\mathrm{O}, \mathrm{S}, \mathrm{Se}) \mathrm{C}_{5}$

$\mathrm{C}_{2}-(\mathrm{O}, \mathrm{S}, \mathrm{Se})_{3}$

$(\mathrm{O}, \mathrm{S}, \mathrm{Se})_{3}-\mathrm{C}_{4}$

$\mathrm{C}_{4}-(\mathrm{O}, \mathrm{S}, \mathrm{Se})_{5}$

$\mathrm{C}_{1}-\mathrm{H}_{6}$

$\mathrm{C}_{2}-\mathrm{H}_{7}$

$\mathrm{C}_{4}-\mathrm{H}_{8}$

$\mathrm{C}_{4}-\mathrm{H}_{9}$

Angles (degrees)

$\mathrm{C}_{1}=\mathrm{C}_{2}-(\mathrm{O}, \mathrm{S}, \mathrm{Se})_{3}$

$(\mathrm{O}, \mathrm{S}, \mathrm{Se})_{5}-\mathrm{C}_{1}=\mathrm{C}_{2}$

$\mathrm{C}_{2}-(\mathrm{O}, \mathrm{S}, \mathrm{Se})_{3}-\mathrm{C}_{4}$

$\mathrm{C}_{4}-(\mathrm{O}, \mathrm{S}, \mathrm{Se})_{5}-\mathrm{C}_{1}$

$(\mathrm{O}, \mathrm{S}, \mathrm{Se})_{3}-\mathrm{C}_{4}-(\mathrm{O}, \mathrm{S}, \mathrm{Se})_{5}$

$\mathrm{C}_{1}=\mathrm{C}_{2}-\mathrm{H}_{7}$

$\mathrm{C}_{2}=\mathrm{C}_{1}-\mathrm{H}_{6}$

$(\mathrm{O}, \mathrm{S}, \mathrm{Se})_{3}-\mathrm{C}_{2}-\mathrm{H}_{7}$

$(\mathrm{O}, \mathrm{S}, \mathrm{Se})_{3}-\mathrm{C}_{4}-\mathrm{H}_{8}$

$(\mathrm{O}, \mathrm{S}, \mathrm{Se})_{3}-\mathrm{C}_{4}-\mathrm{H}_{9}$

$(\mathrm{O}, \mathrm{S}, \mathrm{Se})_{5}-\mathrm{C}_{4}-\mathrm{H}_{8}$

$(\mathrm{O}, \mathrm{S}, \mathrm{Se})_{5}-\mathrm{C}_{4}-\mathrm{H}_{9}$

$(\mathrm{O}, \mathrm{S}, \mathrm{Se})_{5}-\mathrm{C}_{1}-\mathrm{H}_{6}$

$\mathrm{H}_{8}-\mathrm{C}_{4}-\mathrm{H}_{9}$

Angles between bonds

$\mathrm{C}_{1}=\mathrm{C}_{2} /(\mathrm{O}, \mathrm{S}, \mathrm{Se})_{3}-\mathrm{C}_{4}$

$\mathrm{C}_{2}=\mathrm{C}_{1} /(\mathrm{O}, \mathrm{S}, \mathrm{Se})_{5}-\mathrm{C}_{4}$

$(\mathrm{O}, \mathrm{S}, \mathrm{Se})_{3}-\mathrm{C}_{2} / \mathrm{C}_{1}-(\mathrm{O}, \mathrm{S}, \mathrm{Se})_{5}$

$(\mathrm{O}, \mathrm{S}, \mathrm{Se})_{3}-\mathrm{C}_{2} /(\mathrm{O}, \mathrm{S}, \mathrm{Se})_{5}-\mathrm{C}_{4}$

$(\mathrm{O}, \mathrm{S}, \mathrm{Se})_{5}-\mathrm{C}_{1} /(\mathrm{O}, \mathrm{S}, \mathrm{Se})_{3}-\mathrm{C}_{4}$
1.334

1.385

1.385

1.422

1.422

1.073

1.073

1.097

1.084

110.2

110.2

102.3

102.3

107.2

131.8

131.8

117.9

108.8

109.8

108.8

109.8

117.9

112.3

1.337
1.371
1.767
1.829
1.424
1.077
1.075
1.092
1.084

1.336

1.342

1.340

1.339

1.370

1.756

1.754

1.885

1.894

1.756

1.886

1.885

1.963

1.816

1.948

1.943

1.420

1.816

1.812

1.943

1.078

1.079

1.081

1.081

1.075

1.079

1.079

1.087

1.081

1.090

1.089

1.086

1.086

1.086

1.084

111.4

117.0

111.5

119.0

117.3

117.3

117.0

119.3

119.0

86.3

107.7

107.8

82.4

92.6

89.2

119.0

109.6

107.8

92.6

108.6

94.9

108.8

126.1

125.1

124.1

123.6

123.4

119.3

118.6

122.3

126.8

123.2

108.9

110.8

108.9

108.3

110.9

109.4

108.9

108.7

115.0

111.4

114.1

111.5

109.7

109.1

109.7

109.1

118.6

110.5

109.4

108.9

110.3

108.7

117.2

110.6

91.7

91.7

109.0

123.1

123.1

117.9

110.0

108.5

110.0

108.5

117.9

110.7

\begin{tabular}{rrrrrr}
16.7 & 17.0 & 16.0 & 20.2 & 18.7 & 18.7 \\
-16.7 & -20.5 & -21.3 & -20.2 & -20.5 & -18.7 \\
0.0 & -1.5 & -1.3 & 0.0 & 0.2 & 0.0 \\
-17.4 & -21.3 & -21.5 & -21.9 & -21.6 & -20.2 \\
17.4 & 18.5 & 18.1 & 21.9 & 20.9 & 20.2 \\
\hline
\end{tabular}




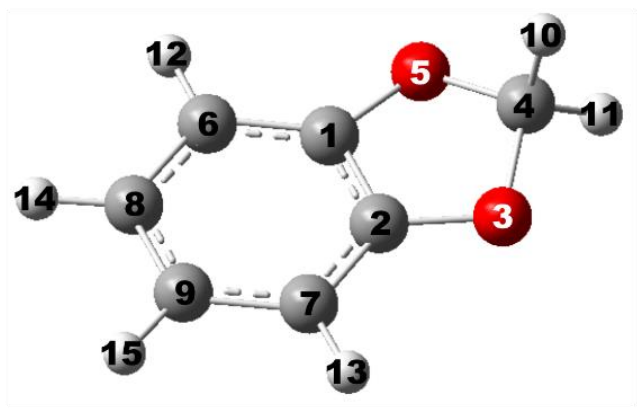

\begin{tabular}{|c|c|c|}
\hline & \\
\hline & CCSD/cc-pVTZ & MP2/cc-pV \\
\hline \multicolumn{3}{|l|}{ Bond lengths (̊̊) } \\
\hline $\mathrm{C}_{1}=\mathrm{C}_{2}$ & 1.389 & 1.390 \\
\hline $\mathrm{C}_{1}-(\mathrm{O}, \mathrm{S}, \mathrm{Se}) \mathrm{C}_{5}$ & 1.372 & 1.374 \\
\hline $\mathrm{C}_{2}-(\mathrm{O}, \mathrm{S}, \mathrm{Se})_{3}$ & 1.423 & 1.374 \\
\hline$(\mathrm{O}, \mathrm{S}, \mathrm{Se})_{3}-\mathrm{C}_{4}$ & 1.423 & 1.427 \\
\hline $\mathrm{C}_{4}-(\mathrm{O}, \mathrm{S}, \mathrm{Se})_{5}$ & 1.423 & 1.427 \\
\hline $\mathrm{C}_{1}-\mathrm{C}_{6}$ & 1.374 & 1.379 \\
\hline $\mathrm{C}_{2}-\mathrm{C}_{7}$ & 1.374 & 1.379 \\
\hline $\mathrm{C}_{4}-\mathrm{H}_{10}$ & 1.094 & 1.094 \\
\hline $\mathrm{C}_{4}-\mathrm{H}_{11}$ & 1.086 & 1.085 \\
\hline $\mathrm{C}_{6}-\mathrm{C}_{8}$ & 1.405 & 1.402 \\
\hline $\mathrm{C}_{7}-\mathrm{C}_{9}$ & 1.405 & 1.402 \\
\hline $\mathrm{C}_{8}-\mathrm{C}_{9}$ & 1.388 & 1.394 \\
\hline $\mathrm{C}_{6}-\mathrm{H}_{12}$ & 1.079 & 1.080 \\
\hline $\mathrm{C}_{7}-\mathrm{H}_{13}$ & 1.079 & 1.080 \\
\hline $\mathrm{C}_{8}-\mathrm{H}_{14}$ & 1.080 & 1.081 \\
\hline $\mathrm{C}_{9}-\mathrm{H}_{15}$ & 1.080 & 1.081 \\
\hline \multicolumn{3}{|l|}{ Angles (degrees) } \\
\hline $\mathrm{C}_{1}=\mathrm{C}_{2}-(\mathrm{O}, \mathrm{S}, \mathrm{Se})_{3}$ & 109.3 & 109.5 \\
\hline$(\mathrm{O}, \mathrm{S}, \mathrm{Se})_{5}-\mathrm{C}_{1}=\mathrm{C}_{2}$ & 109.3 & 109.5 \\
\hline $\mathrm{C}_{2}-(\mathrm{O}, \mathrm{S}, \mathrm{Se})_{3}-\mathrm{C}_{4}$ & 104.3 & 103.5 \\
\hline $\mathrm{C}_{4}-(\mathrm{O}, \mathrm{S}, \mathrm{Se})_{5}-\mathrm{C}_{1}$ & 104.3 & 103.5 \\
\hline$(\mathrm{O}, \mathrm{S}, \mathrm{Se})_{3}{ }_{3} \mathrm{C}_{4}-(\mathrm{O}, \mathrm{S}, \mathrm{Se})_{5}$ & 107.7 & 107.7 \\
\hline $\mathrm{C}_{1}=\mathrm{C}_{2}-\mathrm{C}_{7}$ & 122.1 & 122.1 \\
\hline $\mathrm{C}_{2}=\mathrm{C}_{1}-\mathrm{C}_{6}$ & 122.1 & 122.0 \\
\hline$(\mathrm{O}, \mathrm{S}, \mathrm{Se})_{3}-\mathrm{C}_{2}-\mathrm{C}_{7}$ & 128.5 & 128.4 \\
\hline$(\mathrm{O}, \mathrm{S}, \mathrm{Se})_{3}-\mathrm{C}_{4}-\mathrm{H}_{10}$ & 109.3 & 109.1 \\
\hline$(\mathrm{O}, \mathrm{S}, \mathrm{Se})_{3}-\mathrm{C}_{4}-\mathrm{H}_{11}$ & 109.5 & 109.4 \\
\hline$(\mathrm{O}, \mathrm{S}, \mathrm{Se})_{5}-\mathrm{C}_{4}-\mathrm{H}_{10}$ & 109.3 & 109.1 \\
\hline$(\mathrm{O}, \mathrm{S}, \mathrm{Se})_{5}-\mathrm{C}_{4}-\mathrm{H}_{11}$ & 109.5 & 109.4 \\
\hline$(\mathrm{O}, \mathrm{S}, \mathrm{Se})_{5}-\mathrm{C}_{1}-\mathrm{C}_{6}$ & 128.5 & 128.4 \\
\hline $\mathrm{H}_{10}-\mathrm{C}_{4}-\mathrm{H}_{11}$ & 111.6 & 112.1 \\
\hline
\end{tabular}


Table S3. Continued

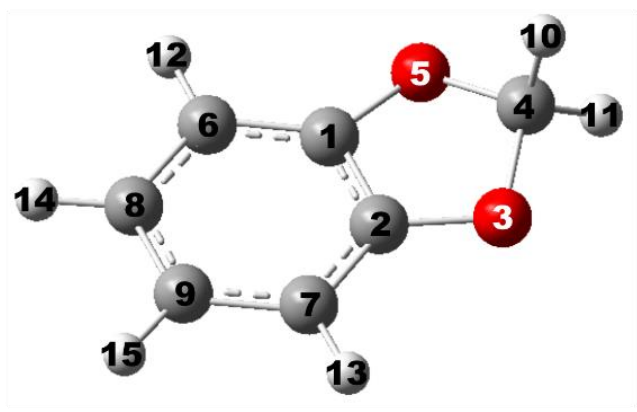

CCSD/cc-pVTZ $\quad$ MP2/cc-pVTZ

Angles (degrees)

$\begin{array}{lll}\mathrm{C}_{1}-\mathrm{C}_{6}=\mathrm{C}_{8} & 116.6 & 116.6 \\ \mathrm{C}_{6}-\mathrm{C}_{8}=\mathrm{C}_{9} & 121.3 & 121.4 \\ \mathrm{C}_{8}-\mathrm{C}_{9}=\mathrm{C}_{7} & 121.3 & 121.4 \\ \mathrm{C}_{9}=\mathrm{C}_{7}-\mathrm{C}_{2} & 116.6 & 116.6 \\ \mathrm{C}_{1}-\mathrm{C}_{6}-\mathrm{H}_{12} & 121.5 & 121.4 \\ \mathrm{C}_{2}-\mathrm{C}_{7}-\mathrm{H}_{13} & 121.5 & 121.4 \\ \mathrm{C}_{8}-\mathrm{C}_{6}-\mathrm{H}_{12} & 121.9 & 122.1 \\ \mathrm{C}_{9}-\mathrm{C}_{7}-\mathrm{H}_{13} & 121.9 & 122.1 \\ \mathrm{C}_{9}-\mathrm{C}_{8}-\mathrm{H}_{14} & 119.6 & 119.5 \\ \mathrm{C}_{8}-\mathrm{C}_{9}-\mathrm{H}_{15} & 119.6 & 119.5\end{array}$

\section{Angles between bonds}

$\mathrm{C}_{1}=\mathrm{C}_{2} /(\mathrm{O}, \mathrm{S}, \mathrm{Se})_{3}-\mathrm{C}_{4}$

13.4

15.0

$\mathrm{C}_{2}=\mathrm{C}_{1} /(\mathrm{O}, \mathrm{S}, \mathrm{Se})_{5}-\mathrm{C}_{4}$

$-13.4$

$-15.0$

$(\mathrm{O}, \mathrm{S}, \mathrm{Se})_{3}-\mathrm{C}_{2} / \mathrm{C}_{1}-(\mathrm{O}, \mathrm{S}, \mathrm{Se})_{5}$

0.0

0.0

$(\mathrm{O}, \mathrm{S}, \mathrm{Se})_{3}-\mathrm{C}_{2} /(\mathrm{O}, \mathrm{S}, \mathrm{Se})_{5}-\mathrm{C}_{4}$

$-13.9$

$-15.6$

$(\mathrm{O}, \mathrm{S}, \mathrm{Se})_{5}-\mathrm{C}_{1} /(\mathrm{O}, \mathrm{S}, \mathrm{Se})_{3}-\mathrm{C}_{4}$

13.9

15.6

$\mathrm{C}_{1}-\mathrm{C}_{6} / \mathrm{C}_{2}-\mathrm{C}_{7}$

180.0

180.0

$\mathrm{C}_{1}-\mathrm{C}_{6} /(\mathrm{O}, \mathrm{S}, \mathrm{Se})_{5}-\mathrm{C}_{4}$

$-15.3$

$\mathrm{C}_{2}-\mathrm{C}_{7} /(\mathrm{O}, \mathrm{S}, \mathrm{Se})_{3}-\mathrm{C}_{4}$

$-14.2$

14.2

15.3

$\mathrm{C}_{1}-\mathrm{C}_{6} / \mathrm{C}_{8}-\mathrm{C}_{9}$

$-0.3$

$-0.4$

$\mathrm{C}_{2}-\mathrm{C}_{7} / \mathrm{C}_{8}-\mathrm{C}_{9}$

$-179.8$

$-179.6$ 


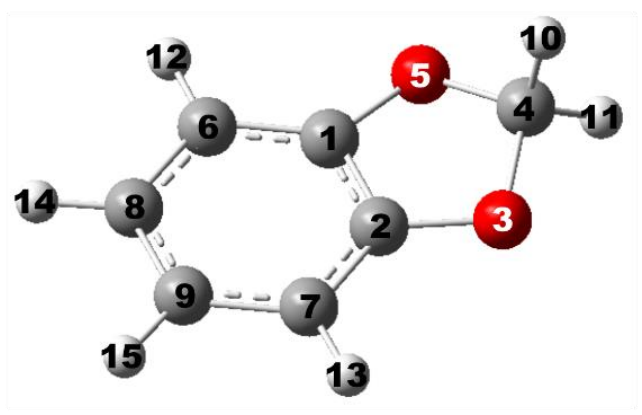

Molecule

Bond lengths (
$\mathrm{C}_{1}=\mathrm{C}_{2}$
$\mathrm{C}_{1}-(\mathrm{O}, \mathrm{S}, \mathrm{Se}) \mathrm{C}_{5}$
$\mathrm{C}_{2}-(\mathrm{O}, \mathrm{S}, \mathrm{Se})_{3}$
$(\mathrm{O}, \mathrm{S}, \mathrm{Se})_{3}-\mathrm{C}_{4}$
$\mathrm{C}_{4}-(\mathrm{O}, \mathrm{S}, \mathrm{Se})_{5}$
$\mathrm{C}_{1}-\mathrm{C}_{6}$
$\mathrm{C}_{2}-\mathrm{C}_{7}$
$\mathrm{C}_{4}-\mathrm{H}_{10}$
$\mathrm{C}_{4}-\mathrm{H}_{11}$
$\mathrm{C}_{6}-\mathrm{C}_{8}$
$\mathrm{C}_{7}-\mathrm{C}_{9}$
$\mathrm{C}_{8}-\mathrm{C}_{9}$
$\mathrm{C}_{6}-\mathrm{H}_{12}$
$\mathrm{C}_{7}-\mathrm{H}_{13}$
$\mathrm{C}_{8}-\mathrm{H}_{14}$
$\mathrm{C}_{9}-\mathrm{H}_{15}$

Angles (degrees)

$\mathrm{C}_{1}=\mathrm{C}_{2}-(\mathrm{O}, \mathrm{S}, \mathrm{Se})_{3}$

$(\mathrm{O}, \mathrm{S}, \mathrm{Se})_{5}-\mathrm{C}_{1}=\mathrm{C}_{2}$

$\mathrm{C}_{2}-(\mathrm{O}, \mathrm{S}, \mathrm{Se})_{3}-\mathrm{C}_{4}$

$\mathrm{C}_{4}-(\mathrm{O}, \mathrm{S}, \mathrm{Se})_{5}-\mathrm{C}_{1}$

$(\mathrm{O}, \mathrm{S}, \mathrm{Se})_{3}-\mathrm{C}_{4}-(\mathrm{O}, \mathrm{S}, \mathrm{Se})_{5}$

$\mathrm{C}_{1}=\mathrm{C}_{2}-\mathrm{C}_{7}$

$\mathrm{C}_{2}=\mathrm{C}_{1}-\mathrm{C}_{6}$

$(\mathrm{O}, \mathrm{S}, \mathrm{Se})_{3}-\mathrm{C}_{2}-\mathrm{C}_{7}$

$(\mathrm{O}, \mathrm{S}, \mathrm{Se})_{3}-\mathrm{C}_{4}-\mathrm{H}_{10}$

$(\mathrm{O}, \mathrm{S}, \mathrm{Se})_{3}-\mathrm{C}_{4}-\mathrm{H}_{11}$

$(\mathrm{O}, \mathrm{S}, \mathrm{Se})_{5}-\mathrm{C}_{4}-\mathrm{H}_{10}$

$(\mathrm{O}, \mathrm{S}, \mathrm{Se})_{5}-\mathrm{C}_{4}-\mathrm{H}_{11}$

$(\mathrm{O}, \mathrm{S}, \mathrm{Se})_{5}-\mathrm{C}_{1}-\mathrm{C}_{6}$

$\mathrm{H}_{10}-\mathrm{C}_{4}-\mathrm{H}_{11}$

\begin{tabular}{llllll}
\hline VII & VIII & IX & X & XI & XII \\
\hline
\end{tabular}

1.390

$1.398 \quad 1.398$

1.403

1.403

1.401

$\begin{array}{lll}1.374 & 1.370 & 1.370 \\ 1.374 & 1.763 & 1.893\end{array}$

1.762

1.763

1.894

1.374

1.831

1.964

1.762

1.893

1.894

1.427

1.421

1.417

1.813

1.945

1.939

$1.379 \quad 1.385$

1.387

1.813

1.807

1.939

1.379

1.389

1.390

1.393

1.395

1.395

1.094

1.092

1.090

1.393

1.393

1.395

1.085

1.085

1.084

1.088

1.087

1.086

1.402

1.397

1.395

1.087

1.087

1.087

1.402

1.396

1.396

1.394

1.393

1.393

1.394

1.394

1.394

1.394

1.394

1.393

1.080

1.081

1.081

1.080

1.081

1.081

1.395

1.394

1.394

1.082

1.082

1.082

1.082

1.082

1.082

1.081

1.081

1.081

1.081

1.081

1.081

1.081

1.081

1.081

1.081

$\begin{array}{rrrrrr}109.5 & 110.5 & 110.6 & 115.9 & 115.7 & 117.4 \\ 109.5 & 115.6 & 117.3 & 115.9 & 117.6 & 117.4 \\ 103.5 & 86.9 & 82.9 & 92.9 & 89.3 & 91.6 \\ 103.5 & 108.3 & 110.1 & 92.9 & 94.9 & 91.6 \\ 107.7 & 107.9 & 107.6 & 108.3 & 108.3 & 108.4 \\ 122.1 & 120.4 & 120.3 & 120.2 & 120.2 & 120.0 \\ 122.1 & 121.4 & 120.3 & 120.2 & 120.2 & 120.0 \\ 128.4 & 129.0 & 129.1 & 123.9 & 124.1 & 122.5 \\ 109.1 & 109.2 & 108.5 & 110.2 & 109.7 & 110.5 \\ 109.4 & 110.3 & 110.5 & 108.8 & 108.8 & 108.4 \\ 109.1 & 109.5 & 110.1 & 110.2 & 110.9 & 110.5 \\ 109.4 & 110.3 & 108.6 & 108.8 & 108.5 & 108.4 \\ 128.4 & 123.0 & 121.6 & 123.9 & 122.4 & 122.5 \\ 112.1 & 111.2 & 111.4 & 110.3 & 110.5 & 110.7\end{array}$


Table S4. Continued

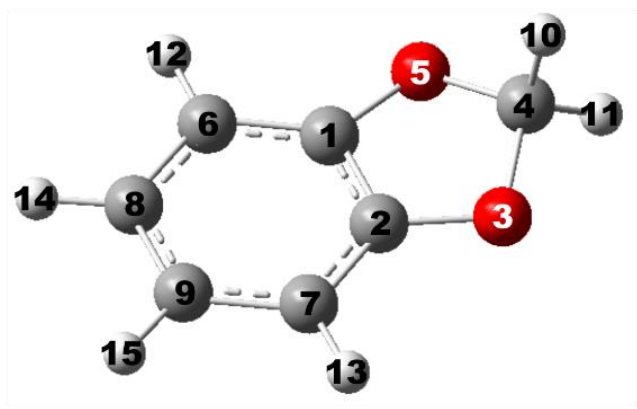

\section{Molecule}

\begin{tabular}{llllll}
\hline VII & VIII & IX & X & XI & XII \\
\hline
\end{tabular}

Angles (degrees)

$\mathrm{C}_{1}-\mathrm{C}_{6}=\mathrm{C}_{8}$

$\mathrm{C}_{6}-\mathrm{C}_{8}=\mathrm{C}_{9}$

$\mathrm{C}_{8}-\mathrm{C}_{9}=\mathrm{C}_{7}$

$\mathrm{C}_{9}=\mathrm{C}_{7}-\mathrm{C}_{2}$

$\mathrm{C}_{1}-\mathrm{C}_{6}-\mathrm{H}_{12}$

$\mathrm{C}_{2}-\mathrm{C}_{7}-\mathrm{H}_{13}$

$\mathrm{C}_{8}-\mathrm{C}_{6}-\mathrm{H}_{12}$

$\mathrm{C}_{9}-\mathrm{C}_{7}-\mathrm{H}_{13}$

$\mathrm{C}_{9}-\mathrm{C}_{8}-\mathrm{H}_{14}$

$\mathrm{C}_{8}-\mathrm{C}_{9}-\mathrm{H}_{15}$

116.6

$118.2 \quad 118.5$

119.4

119.6

119.8

$121.4 \quad 120.8$

120.7

120.4

120.3

120.2

121.4

120.5

120.4

120.3

120.2

$116.6 \quad 118.6$

118.9

119.4

119.6

119.8

$121.4 \quad 120.0$

119.7

120.0

119.9

120.0

$121.4 \quad 120.6$

120.5

120.0

120.

120.0

122.1

121.8

121.7

120.6

120.5

120.2

$122.1 \quad 120.8$

120.5

120.6

120.3

120.2

$119.5 \quad 119.8$

119.9

120.1

120.1

120.1

$119.5 \quad 119.9$

120.0

120.1

120.1

120.1

\section{Angles between bonds}

$\mathrm{C}_{1}=\mathrm{C}_{2} /(\mathrm{O}, \mathrm{S}, \mathrm{Se})_{3}-\mathrm{C}_{4}$

$\mathrm{C}_{2}=\mathrm{C}_{1} /(\mathrm{O}, \mathrm{S}, \mathrm{Se})_{5}-\mathrm{C}_{4}$

15.0

$\begin{array}{rr}15.0 & -1.9 \\ -150 & -21.9\end{array}$

16.5

22.1

20.6

21.8

$-15.0$

$-21.9$

$-24.1$

$-22.1$

$-23.7$

$(\mathrm{O}, \mathrm{S}, \mathrm{Se})_{3}-\mathrm{C}_{2} / \mathrm{C}_{1}-(\mathrm{O}, \mathrm{S}, \mathrm{Se})_{5}$

$\begin{array}{ll}0.0 & -1.0\end{array}$

$-0.3$

0.0

$(\mathrm{O}, \mathrm{S}, \mathrm{Se})_{3}-\mathrm{C}_{2} /(\mathrm{O}, \mathrm{S}, \mathrm{Se})_{5}-\mathrm{C}_{4}$

$-15.6$

$-22.2$

$-23.1$

$-24.1$

0.9

$-21.8$

15.6

19.4

19.7

24.1

$-24.4$

$-23.7$

$\mathrm{C}_{1}-\mathrm{C}_{6} / \mathrm{C}_{2}-\mathrm{C}_{7}$

$\mathrm{C}_{1}-\mathrm{C}_{6} /(\mathrm{O}, \mathrm{S}, \mathrm{Se})_{5}-\mathrm{C}_{4}$

$180.0 \quad 180.0$

180.0

180.0

$23.8 \quad 23.7$

$-15.3 \quad-26.4$

$-30.0$

$-21.4$

180.0

15.3

14.1

13.5

$-23.5$

$-21.5$

$-0.4$

0.0

21.4

19.8

21.5

$\mathrm{C}_{1}-\mathrm{C}_{6} / \mathrm{C}_{8}-\mathrm{C}_{9}$

$-179.6$

$-179.8$

$-179.9$

179.5

0.7

$179.4 \quad 179.3$


Table S5. Coefficients of the Kinetic Energy Functions for Molecules I to XII

$$
g_{44}(x)=c_{0} x^{0}+c_{2} x^{2}+c_{4} x^{4}+c_{6} x^{6} .
$$

\begin{tabular}{|c|c|c|c|c|c|c|}
\hline \multicolumn{7}{|c|}{ Applying CCSD/cc-pVTZ calculated geometrical parameters } \\
\hline & & $\mu_{0}(\mathrm{u})$ & $c_{0}$ & $c_{2}$ & $c_{4}$ & $c_{6}$ \\
\hline I & 1,3-Dioxole & 93.9 & $1.065 \times 10^{-2}$ & $-8.464 \times 10^{-2}$ & $3.416 \times 10^{-1}$ & $-2.152 \times 10^{-1}$ \\
\hline II & 1,3-Oxathiole & 113.4 & $8.819 \times 10^{-3}$ & $-5.177 \times 10^{-2}$ & $2.251 \times 10^{-1}$ & $3.349 \times 10^{-2}$ \\
\hline III & 1,3-Oxaselenole & 144.1 & $6.941 \times 10^{-3}$ & $-3.170 \times 10^{-2}$ & $1.155 \times 10^{-1}$ & $-3.065 \times 10^{-2}$ \\
\hline IV & 1,3-Dithiole & 111.2 & $8.990 \times 10^{-3}$ & $-3.726 \times 10^{-2}$ & $4.205 \times 10^{-2}$ & $-6.393 \times 10^{-2}$ \\
\hline $\mathrm{V}$ & 1,3-Thiaselenole & 129.6 & $7.718 \times 10^{-3}$ & $-2.604 \times 10^{-2}$ & $2.371 \times 10^{-2}$ & $-4.177 \times 10^{-2}$ \\
\hline VI & 1,3-Diselenole & 133.7 & $7.479 \times 10^{-3}$ & $-2.234 \times 10^{-2}$ & $5.128 \times 10^{-3}$ & $-2.304 \times 10^{-2}$ \\
\hline VII & 1,3-Benzodioxole & 145.5 & $6.873 \times 10^{-3}$ & $-5.277 \times 10^{-2}$ & $1.910 \times 10^{-1}$ & $-7.587 \times 10^{-1}$ \\
\hline
\end{tabular}

Applying MP2/cc-pVTZ calculated geometrical parameters

\begin{tabular}{|c|c|c|c|c|c|c|}
\hline & & $\mu_{0}(\mathrm{u})$ & $c_{0}$ & $c_{2}$ & $c_{4}$ & $c_{6}$ \\
\hline I & 1,3-Dioxole & 93.9 & $1.065 \times 10^{-2}$ & $-8.460 \times 10^{-2}$ & $3.416 \times 10^{-1}$ & $-2.135 \times 10^{-1}$ \\
\hline II & 1,3-Oxathiole & 113.3 & $8.828 \times 10^{-3}$ & $-5.185 \times 10^{-2}$ & $2.235 \times 10^{-1}$ & $3.069 \times 10^{-2}$ \\
\hline III & 1,3-Oxaselenole & 143.9 & $6.947 \times 10^{-3}$ & $-3.178 \times 10^{-2}$ & $1.154 \times 10^{-1}$ & $-3.152 \times 10^{-2}$ \\
\hline IV & 1,3-Dithiole & 111.4 & $8.976 \times 10^{-3}$ & $-3.741 \times 10^{-2}$ & $4.263 \times 10^{-2}$ & $-6.463 \times 10^{-2}$ \\
\hline $\mathrm{V}$ & 1,3-Thiaselenole & 129.7 & $7.707 \times 10^{-3}$ & $-2.618 \times 10^{-2}$ & $2.415 \times 10^{-2}$ & $-4.217 \times 10^{-2}$ \\
\hline VI & 1,3-Diselenole & 133.9 & $7.466 \times 10^{-3}$ & $-2.244 \times 10^{-2}$ & $5.257 \times 10^{-3}$ & $-2.334 \times 10^{-2}$ \\
\hline VII & 1,3-Benzodioxole & 145.5 & $6.873 \times 10^{-3}$ & $-5.267 \times 10^{-2}$ & $1.906 \times 10^{-1}$ & $-7.561 \times 10^{-1}$ \\
\hline VIII & 1,3-Benzoxathiole ${ }^{\mathrm{a}}$ & 148.9 & $6.716 \times 10^{-3}$ & $-4.010 \times 10^{-2}$ & $1.140 \times 10^{-1}$ & $-4.212 \times 10^{-1}$ \\
\hline IX & 1,3-Benzoxaselenole ${ }^{\mathrm{a}}$ & 156.7 & $6.382 \times 10^{-3}$ & $-2.451 \times 10^{-2}$ & $2.713 \times 10^{-2}$ & $-6.355 \times 10^{-2}$ \\
\hline $\mathrm{X}$ & 1,3-Benzodithiole & 152.3 & $6.566 \times 10^{-3}$ & $-2.749 \times 10^{-2}$ & $3.722 \times 10^{-2}$ & $-8.536 \times 10^{-2}$ \\
\hline XI & 1,3-Benzothiaselenole $\mathrm{a}^{\mathrm{a}}$ & 159.6 & $6.266 \times 10^{-3}$ & $-2.253 \times 10^{-2}$ & $2.046 \times 10^{-2}$ & $-4.913 \times 10^{-2}$ \\
\hline XII & 1,3-Benzodiselenole & 166.8 & $5.995 \times 10^{-3}$ & $-1.763 \times 10^{-2}$ & $3.876 \times 10^{-3}$ & $-1.330 \times 10^{-2}$ \\
\hline
\end{tabular}

${ }^{\mathrm{a}}$ Estimated. 
Table S6. Comparison of Average Square Deviations (ASD) for the Frequency Fits of 1,3-Dioxole (I)

\begin{tabular}{|c|c|c|c|c|c|c|c|}
\hline \multirow{3}{*}{$\frac{\text { Transition }}{\text { FIR }^{\mathrm{a}}}$} & \multirow{3}{*}{$\frac{\text { Observed }^{\mathrm{a}}}{\mathrm{V}\left(\mathrm{cm}^{-1}\right)}$} & \multicolumn{6}{|c|}{ Experimental fit } \\
\hline & & \multicolumn{3}{|c|}{ This work $^{b}$} & \multicolumn{3}{|c|}{ Literature $^{\mathrm{a}}$} \\
\hline & & $\mathrm{V}\left(\mathrm{cm}^{-1}\right)$ & $\Delta$ & $\Delta^{2}$ & $\mathrm{~V}\left(\mathrm{~cm}^{-1}\right)$ & $\Delta$ & $\Delta^{2}$ \\
\hline $1-2$ & 158.6 & 158.7 & -0.1 & 0.01 & 158.4 & 0.2 & 0.04 \\
\hline $2-3$ & 47.9 & 48.7 & -0.8 & 0.64 & 48.8 & -0.9 & 0.81 \\
\hline $3-4$ & 113.1 & 114.0 & -0.9 & 0.81 & 113.9 & -0.8 & 0.64 \\
\hline $4-5$ & 115.9 & 115.9 & 0.0 & 0.00 & 115.8 & 0.1 & 0.01 \\
\hline $5-6$ & 133.3 & 133.1 & 0.2 & 0.04 & 132.9 & 0.4 & 0.16 \\
\hline $6-7$ & 144.4 & 144.1 & 0.3 & 0.09 & 143.8 & 0.6 & 0.36 \\
\hline $7-8$ & 155.0 & 154.1 & 0.9 & 0.81 & 153.8 & 1.2 & 1.44 \\
\hline $8-9$ & 165.9 & 162.9 & 3.0 & 9.00 & 162.5 & 3.4 & 11.56 \\
\hline $9-10$ & 172.0 & 170.9 & 1.1 & 1.21 & 170.7 & 1.3 & 1.69 \\
\hline $10-11$ & 180.0 & 178.1 & 1.9 & 3.61 & 177.7 & 2.3 & 5.29 \\
\hline $11-12$ & 185.8 & 184.7 & 1.1 & 1.21 & 185.7 & 0.1 & 0.01 \\
\hline $0-3$ & 208.6 & 210.0 & -1.4 & 1.96 & 209.9 & -1.3 & 1.69 \\
\hline $1-4$ & 319.7 & 321.4 & -1.7 & 2.89 & 321.1 & -1.4 & 1.96 \\
\hline $2-5$ & 277.0 & 278.6 & -1.6 & 2.56 & 278.6 & -1.6 & 2.56 \\
\hline \multicolumn{8}{|l|}{ Raman $^{\mathrm{a}}$} \\
\hline $0-2$ & 161.3 & 161.3 & 0.0 & 0.00 & 161.0 & 0.3 & 0.09 \\
\hline $1-3$ & 206.3 & 207.4 & -1.1 & 1.21 & 207.2 & -0.9 & 0.81 \\
\hline $2-4$ & 161.3 & 162.7 & -1.4 & 1.96 & 162.7 & -1.4 & 1.96 \\
\hline $3-5$ & 229.0 & 230.0 & -1.0 & 1.00 & 229.8 & -0.8 & 0.64 \\
\hline $4-6$ & 249.0 & 249.0 & 0.0 & 0.00 & 248.8 & 0.2 & 0.04 \\
\hline $5-7$ & 278.0 & 277.2 & 0.8 & 0.64 & 276.8 & 1.2 & 1.44 \\
\hline $6-8$ & 299.0 & 298.2 & 0.8 & 0.64 & 297.6 & 1.4 & 1.96 \\
\hline $0-4$ & 321.8 & 324.0 & -2.2 & 4.84 & 323.8 & -2.0 & 4.00 \\
\hline $1-2$ & 158.6 & 158.7 & -0.1 & 0.00 & 161.0 & 0.3 & 0.09 \\
\hline $2-3$ & 47.9 & 48.7 & -0.8 & 1.21 & 207.2 & -0.9 & 0.81 \\
\hline $3-4$ & 113.1 & 114.0 & -0.9 & 1.96 & 162.7 & -1.4 & 1.96 \\
\hline $4-5$ & 115.9 & 115.9 & 0.0 & 1.00 & 229.8 & -0.8 & 0.64 \\
\hline $5-6$ & 133.3 & 133.1 & 0.2 & 0.00 & 248.8 & 0.2 & 0.04 \\
\hline $6-7$ & 144.4 & 144.1 & 0.3 & 0.64 & 276.8 & 1.2 & 1.44 \\
\hline SUM: & & & & 35.13 & & & 39.16 \\
\hline ASD: & & & & 1.6 & & & 1.8 \\
\hline
\end{tabular}

${ }^{\mathrm{a}}$ From Reference $9, \mathrm{~g}_{44}(x)=1.406 \times 10^{-2}-8.483 \times 10^{-2} x^{2}+2.468 \times 10^{-1} x^{4}-7.821 \times 10^{-1} x^{6}$; reduced mass: $95.7 \mathrm{u}$; $\mathrm{V}\left(\mathrm{cm}^{-1}\right)=1.59 \times 10^{6} x^{4}-4.18 \times 10^{4} x^{2}+275$.

${ }^{\mathrm{a}} \mathrm{g}_{44}(x)=1.605 \times 10^{-2}-8.464 \times 10^{-2} x^{2}+3.416 \times 10^{-1} x^{4}-2.152 \times 10^{-1} x^{6}$; reduced mass: $93.9 \mathrm{u}$; $\mathrm{V}\left(\mathrm{cm}^{-1}\right)=1.526 \times 10^{6} x^{4}-4.106 \times 10^{4} x^{2}+276$. 


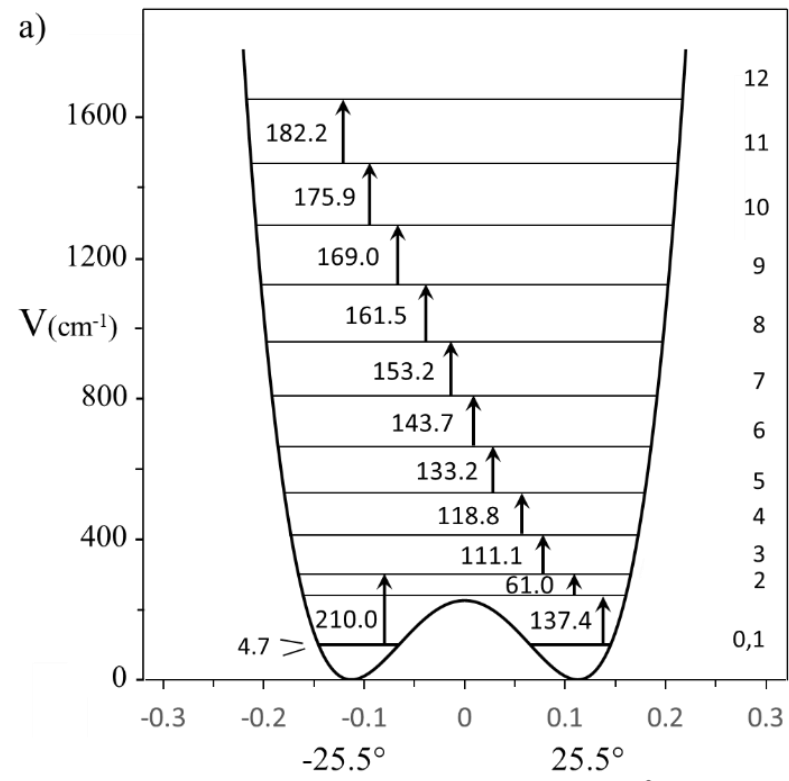

Puckering coordinate, $x(\AA)$

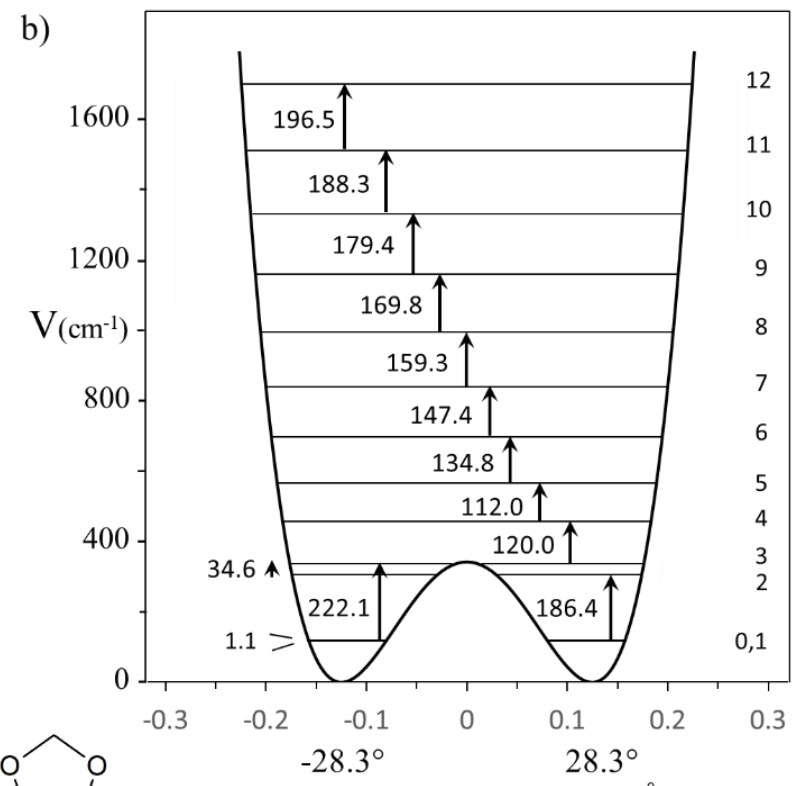

Puckering coordinate, $x(\AA)$

Figure S1. Calculated ring-puckering energy levels from (a) CSSD/cc-pVTZ computations and (b) from MP2/cc-pVTZ computations for 1,3-dioxole (I).

a)

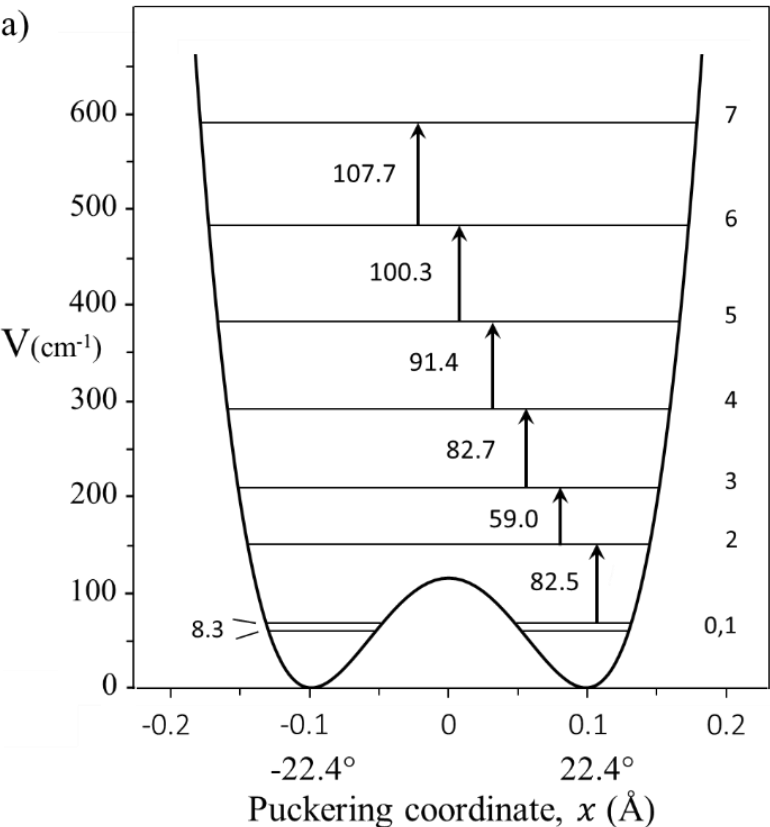

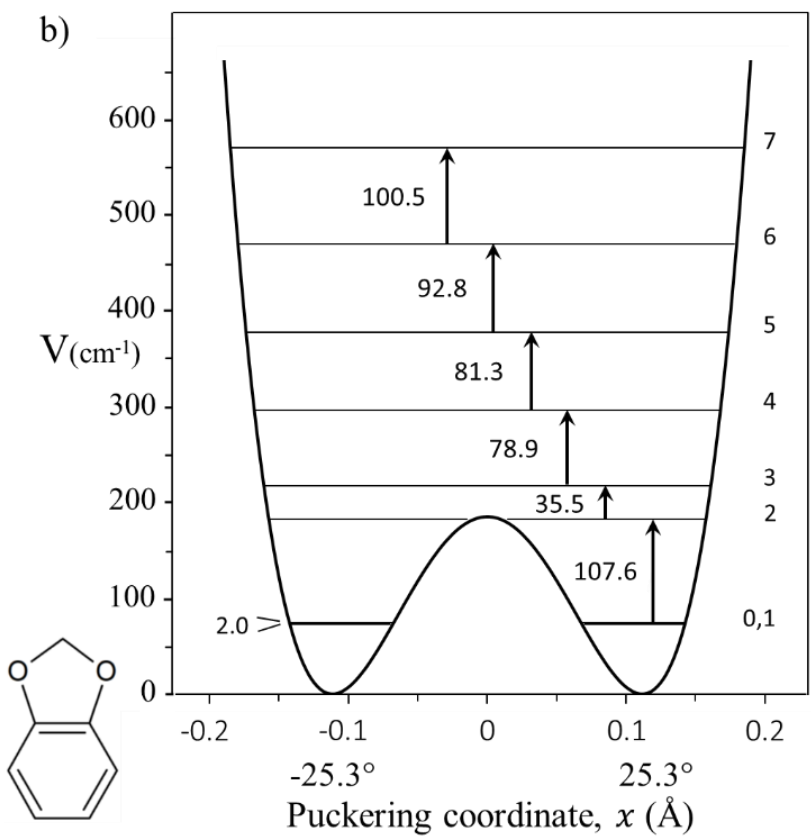

Figure S2. Calculated ring-puckering energy levels from (a) CSSD/cc-pVTZ computations and (b) from MP2/cc-pVTZ computations for 1,3-benzodioxole (VII). 
a)

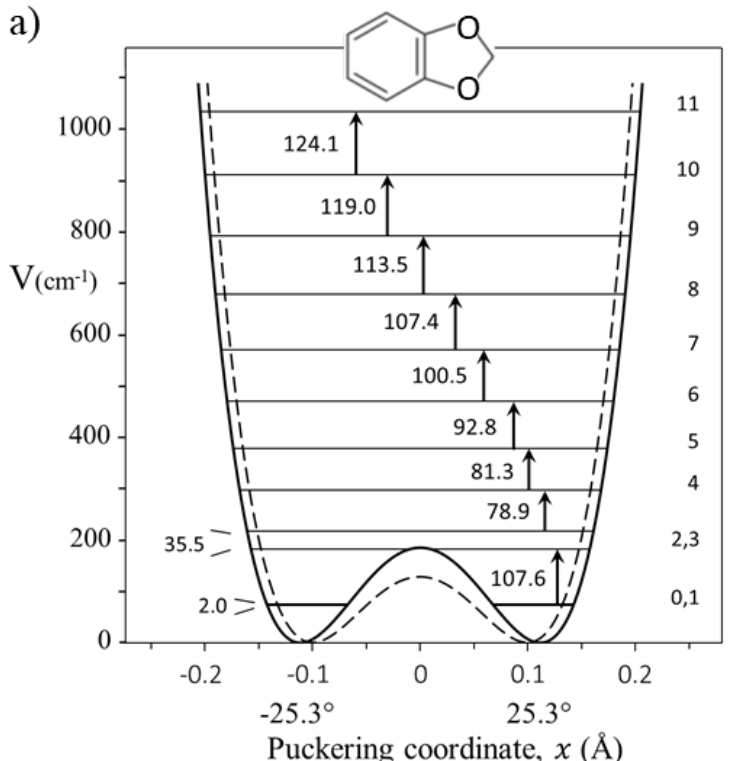

d)

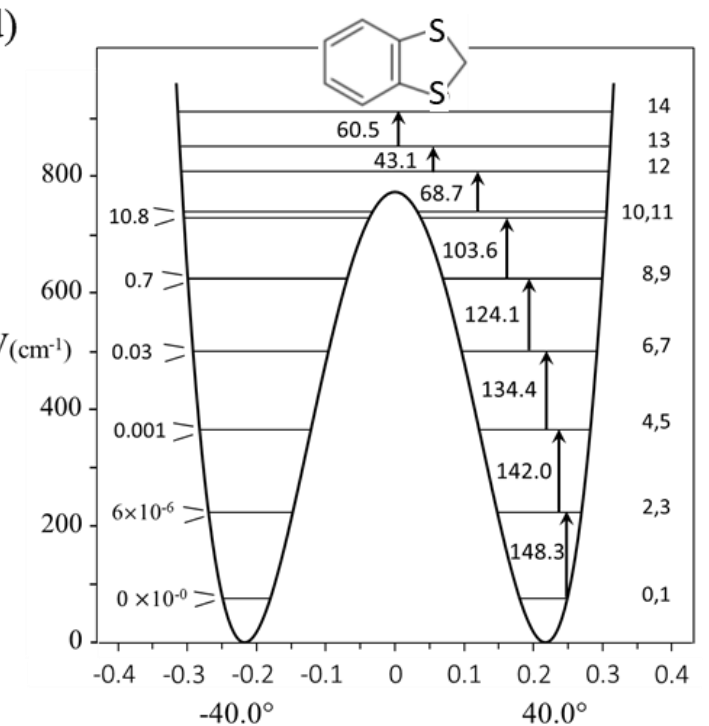

Puckering coordinate, $x(\AA)$ b)

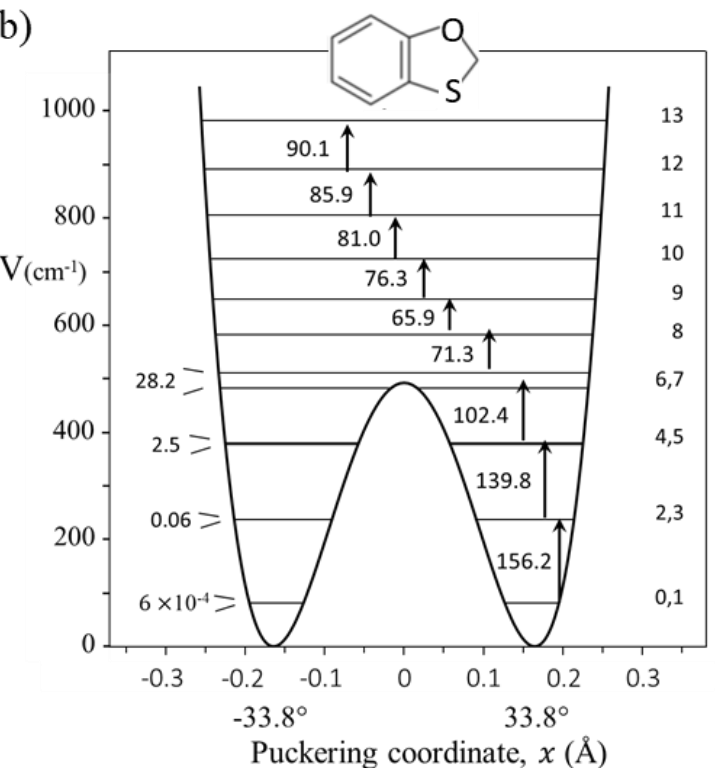

e)

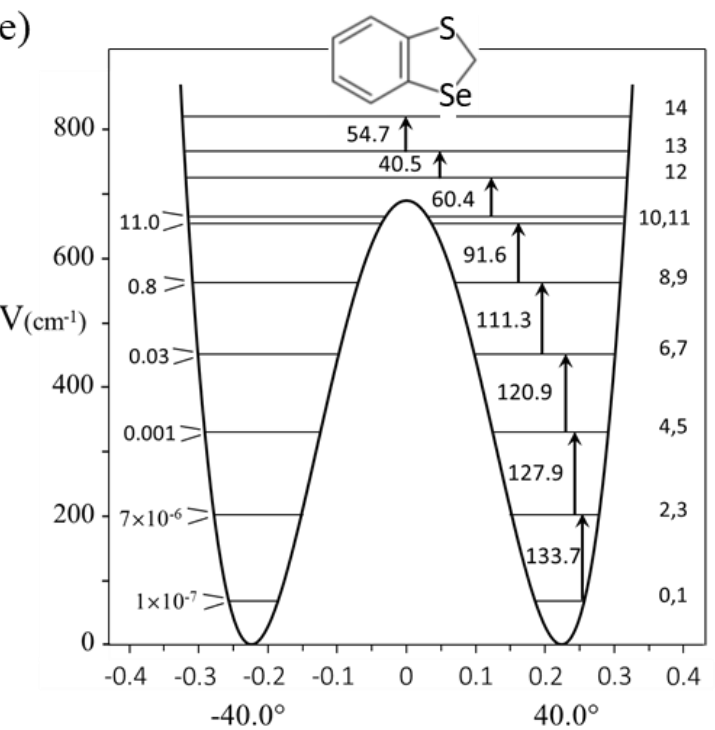

Puckering coordinate, $x(\AA)$

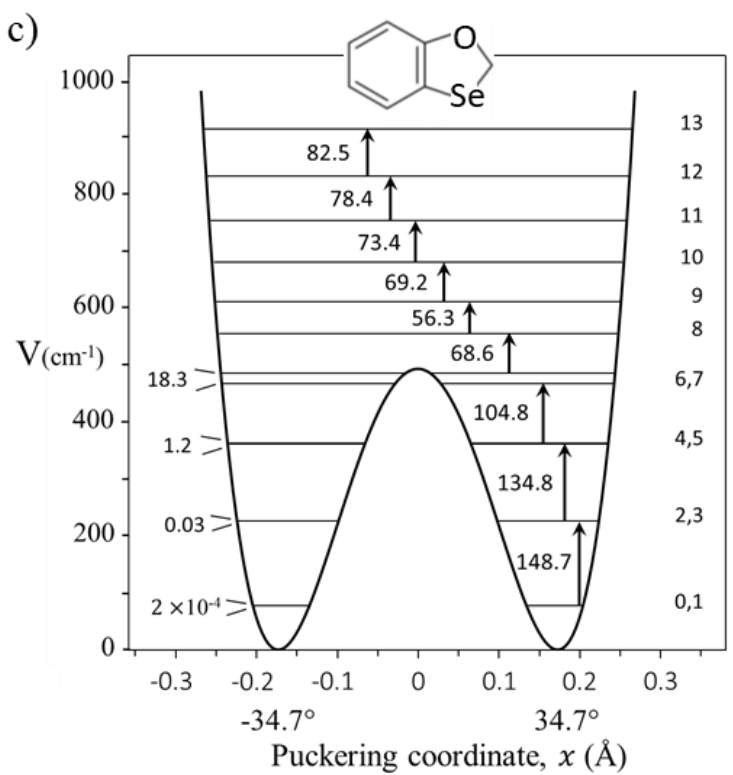

f)

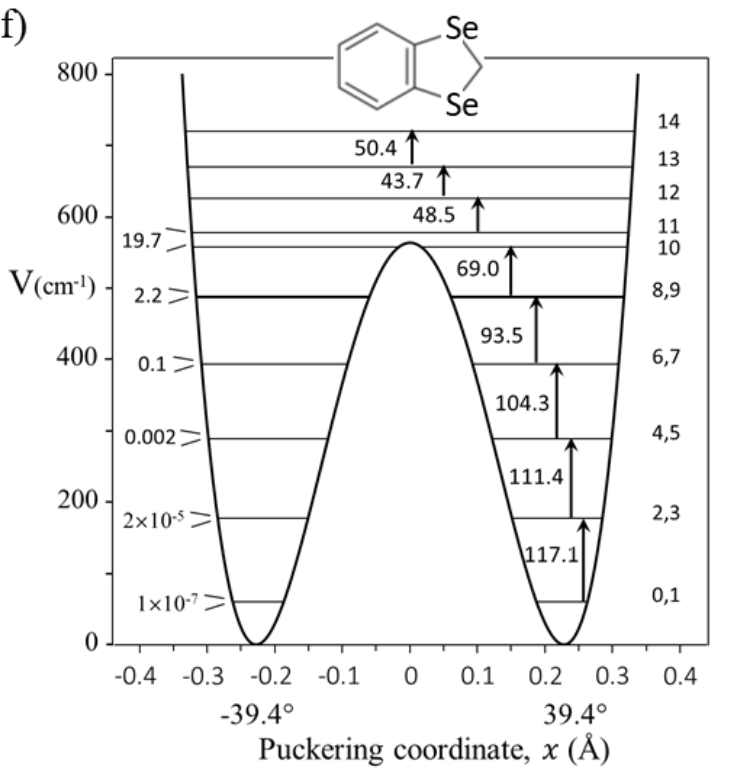

Figure S3. Calculated ring-puckering energy levels from MP2/cc-pVTZ computations for (a) VII, (b) VIII, (c) IX, (d) X, (e) XI and (f) XII. The dashed line in (a) corresponds to the experimental fit for 1,3-benzodioxole (VII). 
Table S7. Calculated Ring-Puckering Transitions $\left(\mathrm{cm}^{-1}\right)$ for 1,3-Oxathiole (II)

\begin{tabular}{|c|c|c|}
\hline \multicolumn{3}{|c|}{ II } \\
\hline \multirow[b]{2}{*}{ Transition } & \multicolumn{2}{|c|}{ Theoretical } \\
\hline & $\mathrm{V}\left(\mathrm{cm}^{-1}\right)^{\mathrm{a}}$ & $\mathrm{V}\left(\mathrm{cm}^{-1}\right)^{\mathrm{b}}$ \\
\hline $0-1$ & 0.2 & 0.01 \\
\hline $1-2$ & 146.1 & 175.2 \\
\hline $2-3$ & 9.3 & 1.1 \\
\hline $3-4$ & 92.6 & 137.2 \\
\hline $4-5$ & 57.1 & 21.5 \\
\hline $5-6$ & 83.7 & 88.7 \\
\hline $6-7$ & 89.5 & 72.6 \\
\hline $7-8$ & 97.9 & 90.3 \\
\hline $8-9$ & 104.5 & 96.5 \\
\hline $9-10$ & 110.5 & 103.6 \\
\hline $10-11$ & 116.0 & 109.5 \\
\hline $11-12$ & 120.9 & 115.0 \\
\hline
\end{tabular}

${ }^{a}$ From CCSD/cc-pVTZ computations.

${ }^{\mathrm{b}}$ From MP2/cc-pVTZ computations.

Table S8. Calculated Ring-Puckering Transitions $\left(\mathrm{cm}^{-1}\right)$ for 1,3-Oxaselenole (III)

\begin{tabular}{|c|c|c|}
\hline \multicolumn{3}{|c|}{ III } \\
\hline \multirow[b]{2}{*}{ Transition } & \multicolumn{2}{|c|}{ Theoretical } \\
\hline & $\mathrm{V}\left(\mathrm{cm}^{-1}\right)^{\mathrm{a}}$ & $\mathrm{V}\left(\mathrm{cm}^{-1}\right)^{\mathrm{b}}$ \\
\hline $0-1$ & 0.1 & 0.01 \\
\hline $1-2$ & 118.6 & 141.9 \\
\hline $2-3$ & 6.3 & 0.6 \\
\hline $3-4$ & 75.7 & 113.5 \\
\hline $4-5$ & 43.3 & 14.4 \\
\hline $5-6$ & 66.2 & 72.4 \\
\hline $6-7$ & 70.5 & 55.3 \\
\hline $7-8$ & 77.5 & 71.3 \\
\hline $8-9$ & 82.9 & 76.1 \\
\hline $9-10$ & 87.7 & 82.0 \\
\hline $10-11$ & 92.2 & 86.8 \\
\hline $11-12$ & 96.2 & 91.2 \\
\hline $12-13$ & 100.0 & 95.3 \\
\hline $13-14$ & 103.5 & 99.1 \\
\hline
\end{tabular}


Table S9. Calculated Ring-Puckering Transitions $\left(\mathrm{cm}^{-1}\right)$ for 1,3-Dithiole (IV)

\begin{tabular}{|c|c|c|}
\hline \multicolumn{3}{|c|}{ IV } \\
\hline \multirow[b]{2}{*}{ Transition } & \multicolumn{2}{|c|}{ Theoretical } \\
\hline & $\mathrm{V}\left(\mathrm{cm}^{-1}\right)^{\mathrm{a}}$ & $\mathrm{V}\left(\mathrm{cm}^{-1}\right)^{\mathrm{b}}$ \\
\hline $0-1$ & 0.01 & 0.0003 \\
\hline $1-2$ & 135.5 & 156.4 \\
\hline $2-3$ & 1.0 & 0.03 \\
\hline $3-4$ & 104.4 & 142.2 \\
\hline $4-5$ & 18.7 & 1.3 \\
\hline $5-6$ & 68.2 & 111.3 \\
\hline $6-7$ & 58.1 & 19.1 \\
\hline $7-8$ & 70.8 & 73.1 \\
\hline $8-9$ & 75.7 & 59.1 \\
\hline $9-10$ & 81.0 & 73.0 \\
\hline $10-11$ & 85.5 & 77.1 \\
\hline $11-12$ & 89.6 & 82.3 \\
\hline $12-13$ & 93.3 & 86.5 \\
\hline $13-14$ & 96.7 & 90.4 \\
\hline
\end{tabular}

${ }^{\mathrm{a}}$ From CCSD/cc-pVTZ computations.

${ }^{\mathrm{b}}$ From MP2/cc-pVTZ computations.

Table S10. Calculated Ring-Puckering Transitions $\left(\mathrm{cm}^{-1}\right)$ for 1,3-Thiaselenole (V)

\begin{tabular}{|c|c|c|}
\hline \multicolumn{3}{|c|}{$\mathbf{V}$} \\
\hline \multirow[b]{2}{*}{ Transition } & \multicolumn{2}{|c|}{ Theoretical } \\
\hline & $\mathrm{V}\left(\mathrm{cm}^{-1}\right)^{\mathrm{a}}$ & $\mathrm{V}\left(\mathrm{cm}^{-1}\right)^{\mathrm{b}}$ \\
\hline $0-1$ & 0.03 & 0.0005 \\
\hline $1-2$ & 108.5 & 128.8 \\
\hline $2-3$ & 1.7 & 0.05 \\
\hline $3-4$ & 77.4 & 114.9 \\
\hline $4-5$ & 22.9 & 2.1 \\
\hline $5-6$ & 54.7 & 83.6 \\
\hline $6-7$ & 53.1 & 23.7 \\
\hline $7-8$ & 60.9 & 58.5 \\
\hline $8-9$ & 65.3 & 54.6 \\
\hline $9-10$ & 69.6 & 62.9 \\
\hline $10-11$ & 73.3 & 66.9 \\
\hline $11-12$ & 76.7 & 71.0 \\
\hline $12-13$ & 79.8 & 74.5 \\
\hline
\end{tabular}


Table S11. Calculated Ring-Puckering Transitions $\left(\mathrm{cm}^{-1}\right)$ for 1,3-Diselenole (VI)

\begin{tabular}{|c|c|c|}
\hline \multicolumn{3}{|c|}{ VI } \\
\hline \multirow[b]{2}{*}{ Transition } & \multicolumn{2}{|c|}{ Theoretical } \\
\hline & $\mathrm{V}\left(\mathrm{cm}^{-1}\right)^{\mathrm{a}}$ & $\mathrm{V}\left(\mathrm{cm}^{-1}\right)^{\mathrm{b}}$ \\
\hline $0-1$ & 0.2 & 0.004 \\
\hline $1-2$ & 82.9 & 106.4 \\
\hline $2-3$ & 7.2 & 0.4 \\
\hline $3-4$ & 51.8 & 86.8 \\
\hline $4-5$ & 36.8 & 9.0 \\
\hline $5-6$ & 50.0 & 55.3 \\
\hline $6-7$ & 53.9 & 38.9 \\
\hline $7-8$ & 58.6 & 52.3 \\
\hline $8-9$ & 62.5 & 55.5 \\
\hline $9-10$ & 66.0 & 59.9 \\
\hline $10-11$ & 69.1 & 63.5 \\
\hline $11-12$ & 72.0 & 66.7 \\
\hline $12-13$ & 74.7 & 69.6 \\
\hline $13-14$ & 77.2 & 72.3 \\
\hline
\end{tabular}

Table S12. Calculated Ring-Puckering Transitions (cm-1) from MP2/cc-pVTZ computations for VII-XII

\begin{tabular}{|c|c|c|c|c|c|c|}
\hline Transition & VII & VIII & IX & $\mathbf{X}$ & XI & XII \\
\hline $0-1$ & 2.0 & 6.E-04 & 2.E-04 & $0 . \mathrm{E}+00$ & 1.E-07 & 1.E-07 \\
\hline $1-2$ & 107.6 & 156.2 & 148.7 & 148.3 & 133.7 & 117.1 \\
\hline $2-3$ & 35.5 & 0.06 & 0.03 & 6.E-06 & 7.E-06 & 2.E-05 \\
\hline $3-4$ & 78.9 & 139.8 & 134.8 & 142.0 & 127.9 & 111.4 \\
\hline $4-5$ & 81.3 & 2.5 & 1.2 & 0.001 & 0.001 & 0.002 \\
\hline $5-6$ & 92.8 & 102.4 & 104.8 & 134.4 & 120.9 & 104.3 \\
\hline $6-7$ & 100.5 & 28.2 & 18.3 & 0.03 & 0.03 & 0.1 \\
\hline $7-8$ & 107.4 & 71.3 & 68.6 & 124.1 & 111.3 & 93.5 \\
\hline $8-9$ & 113.5 & 65.9 & 56.3 & 0.7 & 0.8 & 2.2 \\
\hline $9-10$ & 119.0 & 76.3 & 69.2 & 103.6 & 91.6 & 69.0 \\
\hline $10-11$ & 124.1 & 81.0 & 73.4 & 10.8 & 11.0 & 19.7 \\
\hline $11-12$ & 128.7 & 85.9 & 78.4 & 68.7 & 60.4 & 48.5 \\
\hline $12-13$ & 132.9 & 90.1 & 82.5 & 43.1 & 40.5 & 43.7 \\
\hline $13-14$ & 136.9 & 94.0 & 86.3 & 60.5 & 54.7 & 50.4 \\
\hline
\end{tabular}

